$\Rightarrow$ CANCER GENOMICS

\title{
A multi-layer omics approach to cancer
}

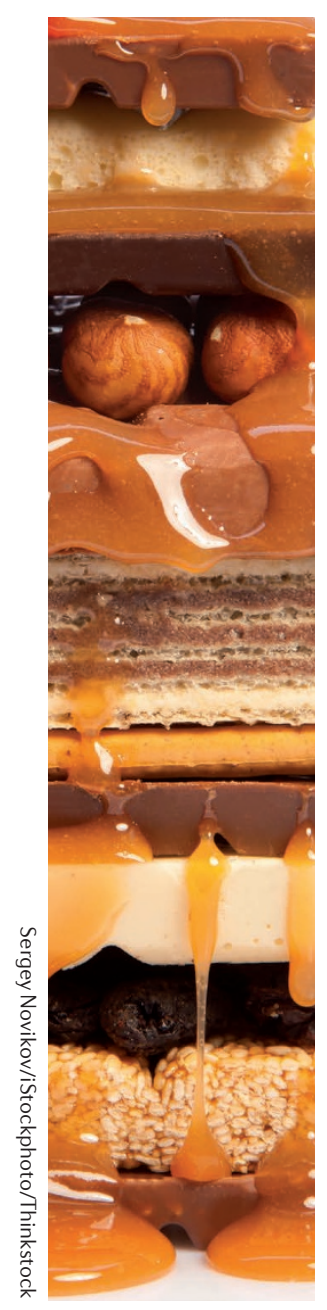

A new study published in the journal Cell integrates proteomic, phosphoproteomic and genomic data to provide important new insights into the biology of the most common form of ovarian cancer.

The Clinical Proteomic Tumor Analysis Consortium (CPTAC) carried out proteomic characterization of tumour samples from high-grade serous carcinomas (HGSCs) that had previously been subject to genomic and transcriptomic analysis. The researchers identified and quantified 9,600 proteins from tumour samples, and selected 3,586 proteins that were present in all samples for further analysis.

They first examined the effects of copy number alterations (CNAs) on protein abundance, as CNAs are a sign of chromosome instability, a hallmark of HGSCs. CNAs in four regions on different chromosomes had the broadest effects on protein expression, altering the abundance of more than 200 proteins in trans. In many cases, transcripts corresponding to the affected proteins were unaltered, suggesting that CNA effects on the proteome can occur at the post-transcriptional level. Notably, the proteins affected by CNAs were enriched for factors involved in cell migration or invasion, and immune regulation, which are processes associated with cancer progression. Analysis of clinical data revealed that the proteome signatures of the four most influential CNA regions were strongly associated with patient survival. Furthermore, several proteins known to be involved in cancer were associated with all four proteome signatures, suggestive of the parallel trans activation by CNAs of multiple pathways that are associated with poor prognosis.

Next, the researchers looked for protein changes associated with homologous recombination deficiency (HRD; defined by mutations in certain genes, including $B R C A 1$ and $B R C A 2$ ), as this feature is associated with response to poly(ADP-ribose) polymerase (PARP) inhibitor therapy and increased survival. They identified a network of 30 proteins exhibiting patterns of co-expression that could be used to distinguish between patients with and without HRD. Further comparitive analyses revealed differences at the level of posttranslational modifications; compared to non-HRD samples, HRD samples displayed decreased acetylation of histone 4 and were enriched for histone deacetylase 1 (HDAC1), which is involved in determining pathways of double-stranded break repair.

Finally, phosphopeptides from 69 tumours were quantified and mapped to pathways from the pathway interaction database (PID). The pathways were then ranked for activity based on phosphoprotein, protein or mRNA levels in samples from shortterm and long-term survivors. Based on the phosphoprotein data, fifteen pathways displayed significantly increased activity in patients with short-term compared to long-term survival. By contrast, only one of these pathways was identified as significantly activated based on transcriptomic data alone.

These experiments illustrate how layering proteomics and genomics an approach termed proteogenomics - can provide additional insights into the pathways that drive cancer and their association with clinical outcomes. Furthermore, the proteomic data generated in this study represent a useful public resource for further studies aimed at improving outcomes in ovarian cancer.

Denise Waldron

ORIGINAL ARTICLE Zhang, H. et al. Integrated proteogenomic characterization of human high-grade serous ovarian cancer. Cell http://dx.doi.org/10.1016/j.cell.2016.05.069 (2016) 